\title{
Systems Engineering perspective to the Integration of the Heating and Current Drive System in the EU DEMO: Analysis of Requirements and Functions
}

\author{
${ }^{a}$ Giovanni Grossetti, ${ }^{b, c}$ Richard Brown, ${ }^{b, d}$ Thomas Franke, ${ }^{a}$ Juergen Gafert, ${ }^{c}$ Tanya Galliara, \\ ${ }^{c}$ Ian Jenkins, ${ }^{c}$ Nicolas Mantel, ${ }^{a}$ Dirk Strauß, ${ }^{e}$ Minh Quang Tran, ${ }^{\mathrm{b}, \mathrm{d}}$ Ronald Wenninger \\ ${ }^{a}$ Karlsruhe Institute of Technology, P.O. Box 3640, D-76021 Karlsruhe, Germany \\ ${ }^{b}$ EUROfusion Programme Management Unit, Boltzmann Str. 2, D-85748 Garching, Germany \\ ${ }^{c}$ Culham Centre for Fusion Energy, Abingdon, OX14 3DB, Oxfordshire, U.K. \\ ${ }^{d}$ Max-Planck-Institut für Plasmaphysik, Boltzmann Str. 2, D-85748 Garching, Germany \\ ${ }^{e}$ Swiss Plasma Center EPFL, CH-1015 Lausanne, Switzerland \\ *Corresponding author: giovanni.grossetti@kit.edu
}

\begin{abstract}
In the framework of the EUROfusion Consortium, the Work Package Heating and Current Drive (WPHCD) is undertaking the development and design of the DEMO Heating and Current Drive (HCD) system, supported by the Work Package Plant Level Systems Engineering, Design Integration and Physics Integration (WPPMI). As the DEMO project is in the Pre-Conceptual phase, its development is dominated by uncertainties on system requirements, and by different DEMO designs and scenarios. In this context, a number of outstanding technology and physics issues, e.g. related to safety, neutronics, performance and reliability, need to be resolved using an integrated approach.

For coping with these boundary conditions, we present an initial analysis of HCD requirements and functions through the development of a System Architecture model, i.e. through describing the HCD system in terms of its functions and their interdependencies. Scope of the work is to address uncertainties i) associated with different reference scenarios (e.g. pulsed and steady-state tokamak) and operational concepts; ii) related to system performances. The assessment is part of integration activities, consisting of both systems engineering methodologies and design analysis, all aiming to ensure consistency in the overall EU DEMO plant design.
\end{abstract}

Keywords: DEMO, Heating and Current Drive, Systems Engineering, Integration.

\section{Introduction}

The EUROfusion consortium agreement is the European body composed of 30 Research Units all around Europe which, on behalf of EURATOM, aims to develop nuclear fusion as a sustainable and reliable source of energy [1]. Under its umbrella, the Power Plant Physics and Technology (PPPT) department is undertaking the pre-conceptual development for the demonstration power plant, DEMO [2], [3]. In the frame of PPPT activities, the Work Package Heating and Current Drive (WPHCD) is in charge of developing the concepts and the technologies for a reliable and efficient Heating and Current Drive (HCD) systems [4], to provide the appropriate power to the Fusion Plasma here called Plasma System and/or to generate current in the Plasma System such that fusion can occur.

Three additional heating methods are presently considered for being used in a DEMO configured as a tokamak, operated as pulsed machine (called DEMO1). These are: Electron Cyclotron (EC) [5], Ion Cyclotron (IC) [6] and Neutral Beam Injection (NBI) [7] systems. In addition, within the scope of WPHCD, technical development in the fields of EC radiofrequency (Gyrotrons) [8] and NBI sources that could be used both in a pulsed tokamak (DEMO1) and in a steady state tokamak (DEMO2) are also assessed. The HCD system must be fully integrated in the machine design and a number of outstanding technologies and physics issues need to be solved consistently to comply with the system requirements. These are related to safety, neutronics, performance RAMI (Reliability, Availability, Maintainability and Inspectability) and recirculating power. Currently the project is in the pre-conceptual phase, dominated by variation in the parameters associated with not-fully-defined design concepts and scenarios. This makes the consolidation of system requirements difficult. System Requirements are defined as statements that transform stakeholder views of desired capabilities into a technical solutions that meets the operational needs [9]; functions are defined as the transformation of input flows to output flows, with defined performance [10]. To improve requirement traceability and facilitate scope change impact study inherent to this phase, we present in this paper the initial development of the HCD System(s) Architecture, i.e. assessing the HCD system from the functional point of view. The work is carried out through making use of a Model-Based Systems Engineering (MBSE) approach, i.e. the formalized application of modeling to support 
system requirements definition, design and analysis activities using SysML ${ }^{\circledR}$ [11]. The scope of the work is twofold: to underline uncertainties i) associated with different reference scenarios and operational concepts and ii) related to system performances. The work we present here is part of integration activities, particularly focusing on interfaces, consisting of both systems engineering methodologies compliant with the standard ISO/IEC/IEEE 15288 [9], applied in aerospace and industries and introduced also in Nuclear Fusion [12], and design analysis all aiming at ensuring consistency in the EU DEMO plant design. The paper is structured as follows: in Chapter 2, we present a first assessment of the identification of stakeholders concerned with the HCD system, and their needs. In Chapter 3, we will introduce the concept of System(s) Architecture, as a methodology for consolidating the framework within which specific design options can be developed, and the concept of Use Case studies. In Chapter 4, we will explore the functions and the functional requirements derived by the preliminary Use Cases analysis. In Chapter 5 we will draw the conclusions.

\section{Assessment of Stakeholder needs}

As first and preliminary step, we have assessed and captured the stakeholder needs for the HCD system. In figure 1 is shown the outcome of the analysis: the light brown boxes represent the stakeholders ("actor"), while the light blue boxes ("usecase") represent their interest with respect to the HCD system. As stakeholders, we have identified bodies like the European Commission that provide the financial support to the project and therefore it is assumed to be concerned with the development costs, and the National Regulator, responsible for licensing the DEMO Plant for operations and, therefore, concerned with ensuring safe operations. Being an integrated system of a complex plant, the HCD will need to interface with a number of other DEMO systems. Since the current organization of the DEMO project within PPPT foresees different Work Packages responsible for the development of specific systems, we have included as possible stakeholders Work Packages responsible for the development of systems the HCD is expected to interface with. In particular: WPPMI for physics requirements through scenarios development, the Breeding Blanket system indicated in figure 1 as Work Package Breeding Blanket (WPBB), the Remote Maintenance system (WPRM), the Diagnostic and Control system (WPDC) and the Safety system (WPSAE). It is important to underline that the interfaces between the HCD system and the aforementioned systems may occur both at physical level, e.g. a mechanical penetration, and at functional level, e.g. contributing to the same function, and that all can play a role in the design and integration of the HCD system into the DEMO plant. Industry is already involved for R\&D in the present phase of the project development and will be involved further in the future for prototyping and testing as well as in the manufacturing process of components. Therefore, possible industrial partners have been included as stakeholders with the interest in understanding the HCD system to the extent required to build the system correctly. Also Supervisory and other Committees are expected to have a similar concern in order to provide a valuable input for a further design development. Finally, we allocated to the Project Management Unit (PMU) the responsibilities of managing design variants both at plant and system level currently under assessments, and developing the design consistently, taking into account all the aspects relevant for a nuclear facility.

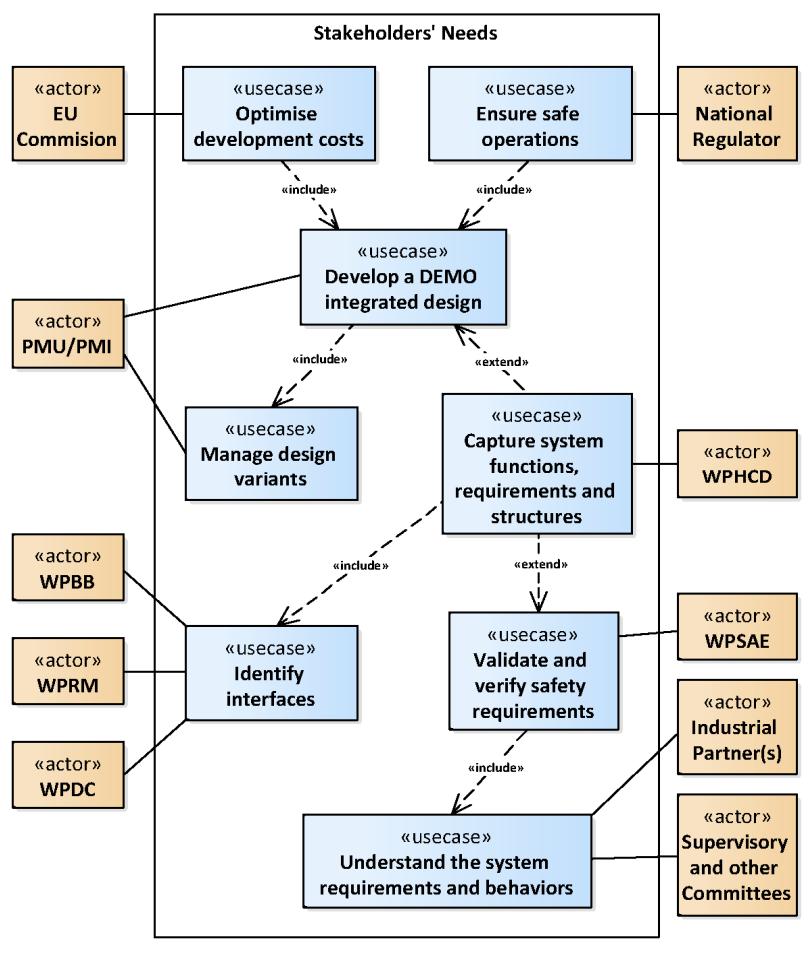

Fig. 1: Use Case diagram for the identification of Stakeholders and their needs with respect to the HCD system.

It is important to remark that the stakeholder' needs reported in figure 1 are a first assessment that will be constantly reassessed and refined, as the development of DEMO and the HCD systems advance.

\section{HCD System(s) Architecture}

In parallel to the analysis of potential HCD stakeholders, we started drafting the foundation of the so-called System(s) Architecture i.e. an abstract, conceptualization-oriented approach to describe the mission and life cycle concepts of the System of Interest (SOI) [9]. Its purpose is to generate system architecture alternatives, to select one or more alternative(s) that frame stakeholder concerns and meet system requirements, and to express this in a set of consistent views. This means to capture the behaviors, i.e. the effect produced when an instance of a complex system is used in its operational environment, of the HCD system with respect to the assumptions over which the DEMO project is based on. Following the approach described in [12], in this first phase we have focused on assessing the HCD system specification, i.e. functions and high level functional requirements, by initiating a Use Case analysis. Use Cases are lists of actions or event steps, 
defining the interactions between an actor and a system, to achieve a goal (in a given context). The actor can be human or another external system. To provide a proper context or boundary where functions and requirements are active, the analysis is framed in a specific DEMO operation state, i.e. the Plasma Operation State (POS), see figure 2 , in which all system required for plasma pulsed operations are active. This is one of the eight DEMO states, the other being the Shutdown State, the Test and Conditioning State, the Stand-by State, the Short, Long and Unscheduled Maintenance States and the Failed State. Here we are assuming the HCD states to be the same as the DEMO states, although this might prove to be not fully correct, as the exploration of specific HCD states has not been initiated yet. For this Use Case study, four actors have been preliminarily identified:

- A National Grid Supplier, assumed to provide the energy to the HCD system for its operation within the considered state

- A Safety System, responsible for ensuring safe operations

- An Integrated Monitor and Control System, responsible to provide the input (parameters) required by HCD for its operations

- A Plasma System, representing the Fusion Plasma.

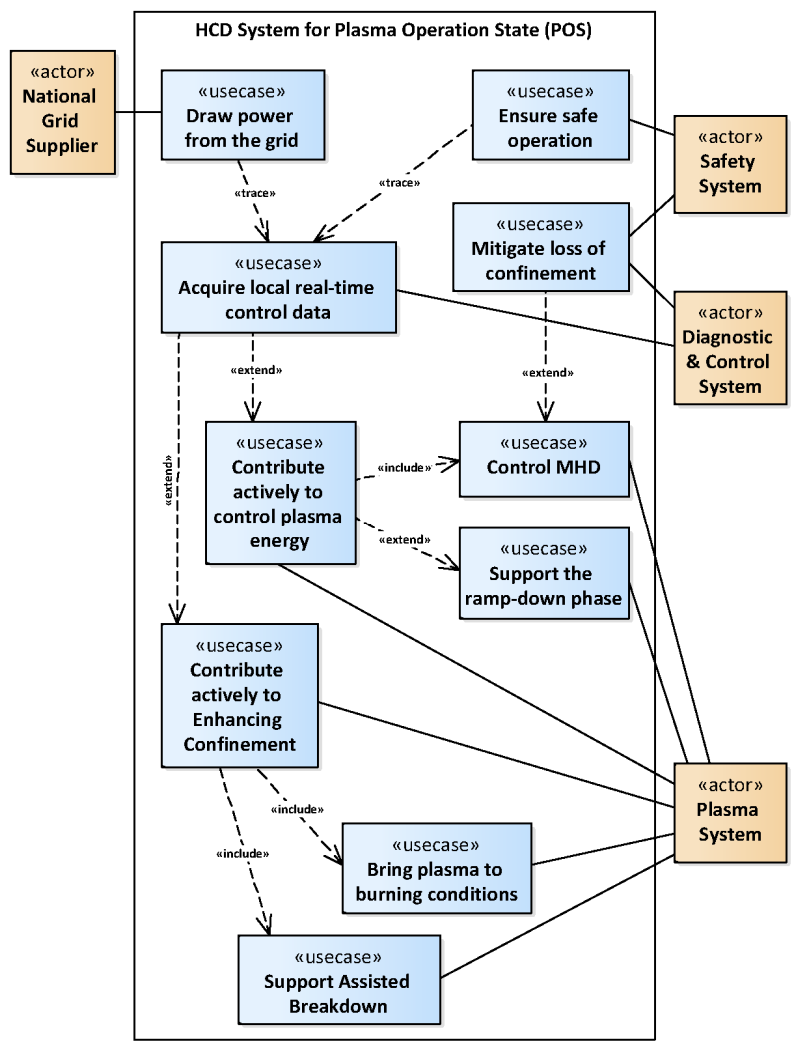

Fig. 2. Use Case diagram for the HCD system for POS

Within the context of POS, a number of Use Cases (represented by light blue boxes) are identified. Each Use Case represents a functionality of the HCD system can be used for during POS. Relationships between Use Cases have also been established. More specifically:

- Include relationship, used when a piece of functionality may be split from the main use case (e.g. Contribute actively to enhance confinement include Bring plasma to burning conditions).

- Extend relationship, used when the functionality of a Use Case may change, depending on what happens when the System is running. An example is Mitigate loss of confinement extends to Control of MHD.

- Trace relationships, used when the functionality is limited by another use case

In figure 2, we show the Use Case diagram for the HCD system for POS. It is important to stress that this representation is still preliminary and due to the high level of uncertainties the use cases need still to be confirmed. In this diagram, two Use Cases are particularly important: Contribute actively to Enhancing Confinement and Contribute actively to control Plasma Energy. These two Use Cases represent in fact the main purposes a HCD system is required for to be integrated into a nuclear fusion power plant. In the following Chapter we will detail the analysis through a first definition of the HCD functions and top level functional requirements.

\section{HCD Functions and functional requirements}

The Use Case study presented in the previous chapter provides the framework for capturing functions and functional requirements (see also [13]).

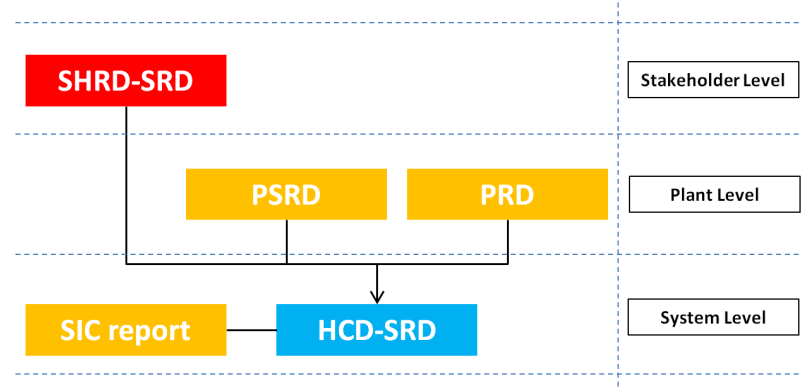

Fig. 3. Top-Down approach followed for the HCD system requirements elicitation

Table 1. Preliminary SBS of the HCD system.

\begin{aligned} & \hline Function Category Purpose \\ & \hline Safety $\begin{array}{l}\text { To meet safety regulations and } \\ \text { requirements } \\ \text { Control }\end{array} \\ &$ To allow for plasma control \\ & (e.g. instability mitigation) \\ & To enhance plasma \\ & performances (e.g. to favor \\ & L-H transition) \\ & Functions shared with other \\ & systems \end{aligned}

The exercise has been carried out following two paths: firstly, in a typical top-down approach, i.e. starting from high level documents like the Stakeholder Requirement Document (SHRD), the Plant Requirement Document (PRD), the Plant Safety Requirement Document (PSRD) and other Safety Important Component (SIC) documents, and propagating functions and requirements 
to the HCD system level (see figure 3). Secondly, exploring the functions starting from the ITER HCD system, that could be allocated in the frame of the specific (not comprehensive) Use Cases reported in figure 2 .

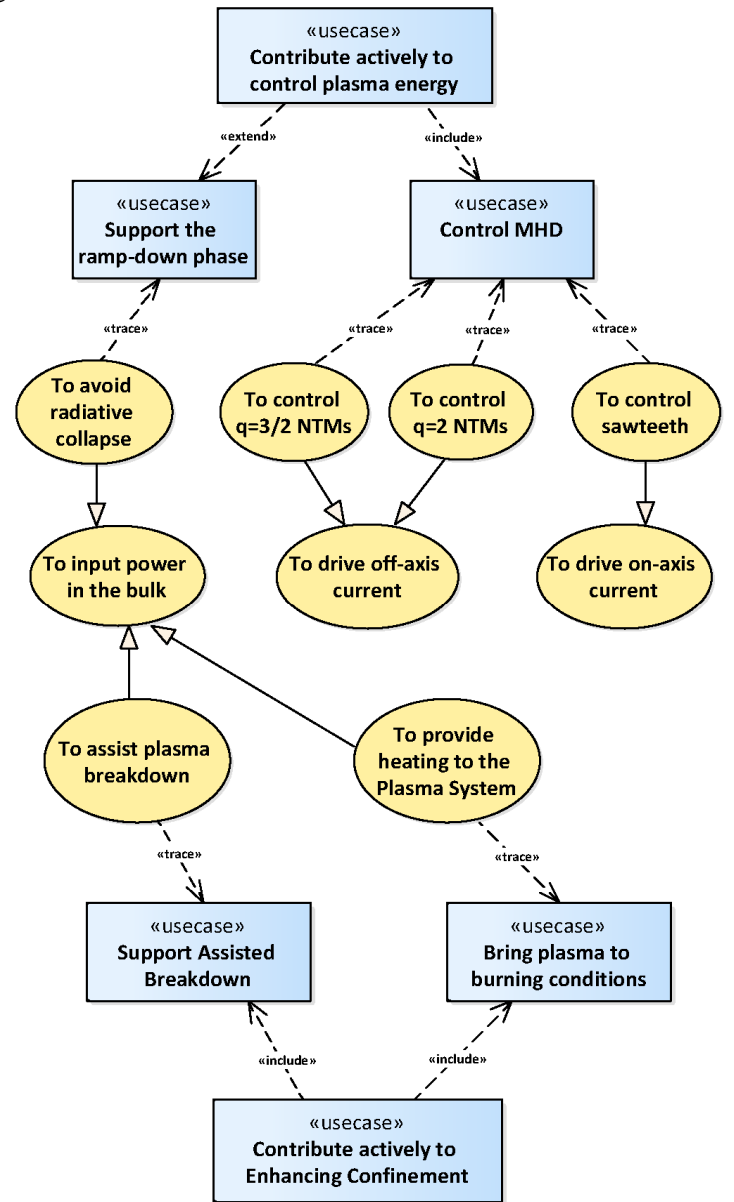

Fig. 4. Relationships between the Use Cases considered in the analysis and main functions for the HCD system

While performing this exercise, functions have been grouped under four different categories, as indicated in table 1 , where each category of functions is associated to a purpose. This approach allowed us to set up a preliminary System Breakdown Structure (SBS) of the HCD system. In figure 4 we present the result of this approach, by showing the functions, represented by yellow circles, and their relationships with the two Use Cases considered, shown with light blue boxes, i.e. Contribute actively to Enhancing Confinement and Contribute actively to control Plasma Energy. The functions falling within these Use Cases are called Burn Functions (associated to Contribute actively to Enhancing Confinement) and Control Functions (associated to Contribute actively to control Plasma Energy). It is important to underline that figure 4 refers to a group of functions and use cases valid for a specific DEMO state assumed valid also for the HCD system, i.e. as mentioned previously POS. Similar exercises need to be carried out also for the other 7 machine states, in order to explore the HCD functions completely. As the MBSE activities have only been initiated recently, we have decided to focus on the POS state as a representative example for showing the benefits of such a Systems Engineering approach in support of design and development of complex systems, like the DEMO HCD. As mentioned at the beginning of this chapter, also functional requirements for the HCD system in the context of the assessed Use Case have been captured following again a Top-Down approach. These are reported in figure 5 and figure 6 as green boxes.
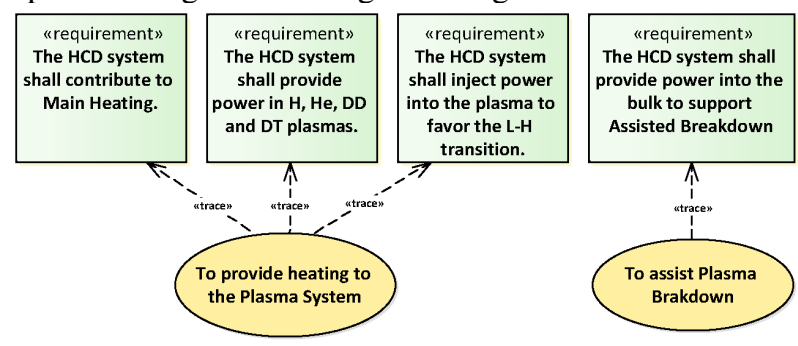

Fig. 5. Requirements elicited for Burn Functions
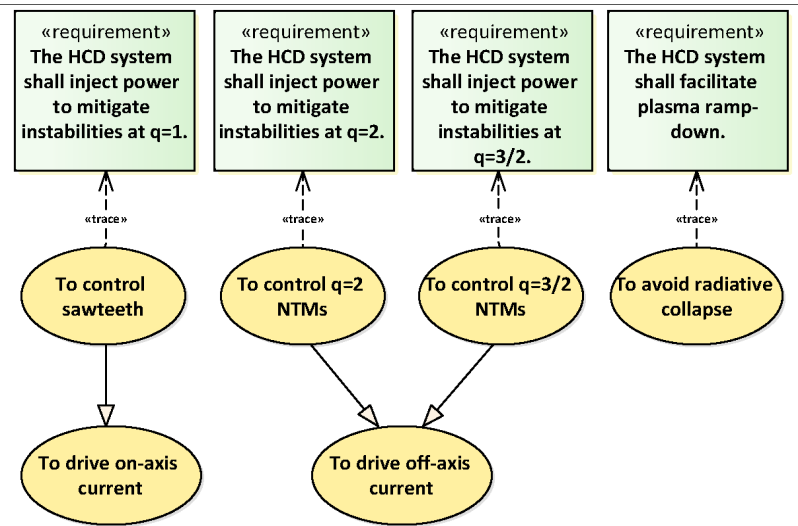

Fig. 6. Requirements elicited for Control Functions

The exercise consisted in capturing then requirements necessary to fulfill the identified functions. This has been done for requirements relevant to safety, burn and control functions, although due to space limitations we show here only the requirements for burn and control. It is important to note that not all the functions identified so far have been consolidated yet and presently they represent a potential scope for the HCD system. This is particularly valid for both burn and control functions, as the amount of power that the HCD system will have to couple to the plasma is not clear yet. In addition, functions like To mitigate disruptions have not been allocated to any requirement as the capturing is still ongoing and did not reach a sufficient maturity level yet. However, despite the still floating requirements, the exercise that we have performed provided a first scheme for a potential HCD system relevant for DEMO, that will be refined as more information from physics will be made available.

\section{Conclusions}

The complexity of the DEMO project, an integrated system composed of several interdependent parts, requires careful design and integration studies. In particular, assessing interfaces results in a difficult task as DEMO is in the Pre-Conceptual phase where design parameters are still fluctuating. In this framework the development of the HCD system becomes a tough task, due to the risks associated with evolving requirements inherent with the current project phase. To facilitate the 
development of the HCD system, in the Systems Engineering framework being implemented by the PPPT department we have applied MBSE methodologies with the aim of a) developing the HCD System(s) Architecture and b) to define a systematic approach to record and assess the impact of requirement evolution. The outcomes presented in this paper are resulting from the cooperation between the Systems Engineering areas of WPHCD and WPPMI focusing on a functional assessment of the HCD system, with the objective to support the design and integration activities. With respect to the HCD system as for many if not all other system in DEMO, the Systems Engineering tasks aim at: managing incomplete and evolving requirements from all project actors. This supports the evaluation of technologies and design options against clear criteria by addressing the HCD system behavior [10] in a systematic approach in a language common to all actors, rather than developing its physical attributes. The exercise consisted in the formalized application of modelling to support the capturing of system requirements and functions from the beginning of the DEMO project (Pre-Conceptual phase). In addition, the Top-Down approach, whose benefits have been proven in a System Architecture analysis for the Breeding Blanket system, has been followed here allowing the necessary consistency with high level (plant) requirements. This is particularly important due to the early phase of the project, in order to define the suitable parameter space where detail design can be developed by engineering specialists. As next steps, we will include in the model the input from a) the Physics analysis by WPPMI and b) the interfaces with WPDC and c) we will allocate HCD functions to the physical system. The results of this study will be incorporated into the Plant Architectural model, together with a similar analysis carried out for the Breeding Blanket and the Remote Maintenance systems. Similar activities are planned to start also for Diagnostic and Control, Vacuum Vessel and Magnet systems in the near future, in order to provide a better definition of system boundaries and interface requirements.

\section{Acknowledgments}

This work has been carried out within the framework of the EUROfusion Consortium and has received funding from the EURATOM research and training programme 2014-2018 under grant agreement No 633053. The views and opinions expressed herein do not necessarily reflect those of the European Commission.

\section{References}

[1] F. Romanelli, P. Barabaschi, D. Borba, G. Federici,

L. Horton, R. Neu et al., "A roadmap to the realization of fusion energy" EFDA pub. 2012, https://www.eurofusion.org/wpcms/wpcontent/uploads/2013/01/JG12.356-web.pdf

[2] G. Federici, C. Bachmann, L. Barucca, W. Biel, L. V. Boccaccini, F. Cismondi, et al., "DEMO Design Activity in Europe: Progress and Updates", $13^{\text {th }}$ International Symposium on Fusion Nuclear Technology
(ISFNT), September $25^{\text {th }}-29^{\text {th }}, 2017$, Kyoto (Japan)

[3] M. Coleman, F. Maviglia, C. Bachmann, J. Anthony,

G. Federici, M. Shannon et al., "On the EU approach for DEMO architecture exploration and dealing uncertainties", Fus. Eng. \& Des. 109-111 (2016) 1158-1162

[4] M. Q. Tran, T. Franke, G. Granucci, G. Grossetti, J. Jelonnek, I. Jenkins et al. ,R\&D in the frame of the PPPT Heating and Current Drive for EU DEMO“, $22^{\text {nd }}$ Radiofrequency Power in Plasma Topical Conference (RFPPC), May $30^{\text {th }}-$ June $2^{\text {nd }}, 2017$, Aix en Provence (France)

[5] S. Garavaglia, G. Aiello, S. Alberti, K. Avramidis, A. Bruschi, I. G. Chelis et al., "EU DEMO EC system preliminary conceptual design", $13^{\text {th }}$ International Symposium on Fusion Nuclear Technology (ISFNT), September $25^{\text {th }}-29^{\text {th }}, 2017$, Kyoto (Japan)

[6] A. Bader, G. Bosia, A Messiaen, R. Ragona, A. Garcia, U. Fisher et al., ,Integrating a distributed antenna DEMO: requirements and challenges“, in press on Fus. Eng. \& Des.

[7] P. Sonato, P. Agostinetti, T. Bolzonella, F. Cismondi, U. Fantz, A. Fassina et al., "Conceptual design of the DEMO neutral beam injectors: main developments and R\&D achievements", Nucl. Fusion 57 (2017) 056026

[8] J. Jelonnek, G. Aiello, S. Alberti, K. Avramidis, A. Bertinetti, A. Bruschi et al., "European Research Activities Towards a Future DEMO Gyrotron", $10^{\text {th }}$ International Workshop "Strong Microwaves and Terahertz Waves: Sources and Applications", July $17^{\text {th }}$ $22^{\text {nd }}, 2017$, Nizhny Novgorod - Moscow (Russia)

[9] ISO/IEC/IEEE. 2015. Systems and Software Engineering - System Life Cycle Processes. Geneva, Switzerland: International Organization for Standardization (ISO)/International Electrotechnical Commission (IEC)/Institute of Electrical and Electronics Engineers (IEEE). ISO/IEC/IEEE 15288:2015 (E).

[10] BKCASE Editorial Board. 2017. The Guide to the Systems Engineering Body of Knowledge (SEBoK), v. 1.8. R.D. Adcock (EIC). Hoboken, NJ: The Trustees of the Stevens Institute of Technology. www.sebokwiki.org.

[11] P. Pearce, S. Friedenthal, "A practical approach for modelling submarine subsystem architecture in SysML", Submarine Institute of Australia Science, Technology \& Engineering Conference 2013

[12] D. Wolff, R. Brown, P. Curson, R. Ellis, T. Galliara, M. Harris, "Early lessons from the application of Systems Engineering at UKAEA", $27^{\text {th }}$ IEEE Symposium On Fusion Engineering, 4-8 June 2017 Shanghai (China)

[13] R. Wenninger, R. Kembleton, C. Bachmann, W. Biel, T. Bolzonella, S. Ciattaglia et al., „The physics and technology basis entering European system code studies for DEMO“, Nucl. Fusion 57 (2017) 016011 